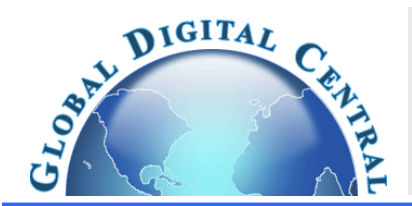

Frontiers in Heat and Mass Transfer

\title{
EFFECT OF CHEMICAL REACTION AND RADIATION ON UNSTEADY CONVECTIVE HEAT AND MASS TRANSFER FLOW OF A VISCOUS FLUID IN A VERTICAL WAVY CHANNEL WITH OSCILLATORY FLUX AND HEAT SOURCES
}

\author{
P.V.S. Kamalakar ${ }^{\mathrm{a}, *}$, R. Raghavender Rao ${ }^{\mathrm{a}}$, D.R.V. Prasada Rao ${ }^{\mathrm{b}}$ \\ ${ }^{a}$ Department of Mathematics, K.L.University, Greenfields, Vaddeswaram, Andhra Pradesh., India \\ ${ }^{b}$ Department of Mathematics, SK University, Ananthapuramu, Andhra Pradesh., India
}

\begin{abstract}
In this paper we discuss the effect of chemical reaction and thermal radiation on unsteady free convective heat and mass transfer flow through a porous medium in a vertical wavy channel. The unsteadiness in the flow is due to the oscillatory flux in the flow region. The coupled equations governing the flow, heat and mass transfer have been solved by using a perturbation technique with the slope $\delta$ of the wavy wall as the perturbation parameter. The expression for the velocity, the temperature, the concentration, the rate of heat and mass transfer are derived and are analyzed for different variations of the governing parameters $\mathrm{G}, \mathrm{R}, \mathrm{M}, \mathrm{D}^{-1}, \beta, \mathrm{N}, \mathrm{N}_{1}, \alpha, \gamma, \mathrm{k}, \mathrm{x}$ and $\mathrm{t}$.

Keywords: Heat \& Mass Transfer, Wavy channel, Oscillatory Flux, Chemical Reaction, Radiation and Heat source
\end{abstract}

\section{INTRODUCTION}

Coupled heat and mass transfer phenomenon in porous media is gaining attention due to its interesting applications. The flow phenomenon is relatively complex rather than that of the pure thermal convection process. Underground spreading chemical wastes and other pollutants, grain storage, evaporation cooling and solidification are the few other application areas where the combined thermo-solutal natural convection in porous media are observed .Combined heat and mass transfer by free convection under boundary layer approximations has been studied by Bejan and Khair (1985), Lai and Kulacki (1991) and Murthy and Singh(1990).Coupled heat and mass transfer by mixed convection in Darcian fluid-saturated porous media has been analyzed by Lai (1971). The free convection heat and mass transfer in a porous enclosure has been studied recently by Angirasa et al. (1997). The combined effects of thermal and mass diffusion in channel flows has been studied in recent times by a few authors, notably Nelson and Wood $(1986,1989)$, Lee at al. (1982).

In recent years, energy and material saving considerations have prompted an expansion of the efforts at producing efficient heat exchanger equipment through augmentation of heat transfer. It has been established that channels with diverging - converging geometries augment the transportation of heat transfer and momentum. As the fluid flows through a tortuous path viz., the dilated - constricted geometry, there will be more intimate contact between them. The flow takes place both axially (primary) and transversely (secondary) with the secondary velocity being towards the axis in the fluid bulk rather than confining within a thin layer as in straight channels. Hence it is advantageous to go for converging - diverging geometries for improving the design of heat transfer equipment. Vajravelu and Nayfeh (1981) have investigated the influence of the wall waviness on friction and pressure drop of the generated coquette flow. Vajravelu and Sastry (1978) have analyzed the free convection heat transfer in a viscous, incompressible fluid confined between long vertical wavy walls in the presence of constant heat source. Vajravelu and Debnath have extended this study to convective flow in a vertical wavy channel in four different geometrical configurations. This problem has been extended to the case of wavy walls by McMichael and Deutsch (1984), Deshikachar et al (1985), Rao et al (1983) and Sree Ramachandra Murthy (1992). Hyan Goo Kwon et al(2008) have analyzed that the Flow and heat/mass transfer in a wavy duct with various corrugation angles in two dimensional flow regimes. Comini et al (2002) have analyzed the Convective heat and mass transfer in wavy finned-tube exchangers.

In many chemical engineering processes, there does occur the chemical reaction between a foreign mass and the fluid in which the plate is moving. These processes take place in numerous industrial applications viz., polymer production, manufacturing of ceramics or glassware and food processing. Das et al(1994) have studied the effects of mass transfer on flow past an impulsively started infinite vertical plate with constant heat flux and chemical reaction. Muthukumaraswamy (2003) has studied the effects of reaction on a long surface with suction. Recently Gnaneswar (2008) has studied radiation and mass transfer on an unsteady two-dimensional laminar convective boundary layer flow of a viscous incompressible chemically reacting fluid along a semi-infinite vertical plate with suction by taking into account the effects of viscous dissipation.

The present trend in the field of chemical reaction analysis is to give a mathematical model for the system to predict the reactor performance. A large amount of research work has been reported in this field. In particular the study of heat and mass transfer with chemical reaction is of considerable importance in chemical and hydrometallurgical industries. Chemical reaction can be codified as either heterogeneous or homogeneous processes. This depends on whether they occur at an interface or as a single phase volume reaction. Frequently the 
transformations proceed in a moving fluid, a situation encountered in a number of technological fields. A common area of interest in the field of aerodynamics is the analysis of thermal boundary layer problems for two dimensional steady and incompressible laminar flow passing a wedge. Simultaneous heat and mass transfer from different geometrics embedded in a porous media has many engineering and geophysical application such as geothermal reservoirs, drying of porous solids thermal insulation, enhanced oil recovery, packed-bed catalytic reactors, cooling of nuclear reactors, and underground energy transport. A very significant area of research in radioactive heat transfer, at the present time is the numerical simulation of combined radiation and convection/conduction transport processes. The effort has arisen largely due to the need to optimize industrial system such as furnaces, ovens and boilers and the interest in our environment and in no conventional energy sources, such as the use of salt-gradient solar ponds for energy collection and storage. In particular, natural convection induced by the simultaneous action of buoyancy forces resulting from thermal and mass diffusion is of considerable interest in nature and in many industrial applications such as geophysics, oceanography, drying process, solidification of binary alloy and chemical engineering. Kandaswamy et al. (2006) have discussed the effects of chemical reaction, heat and mass transfer on boundary layer flow over a porous wedge with heat radiation in the presence of suction or injection. Madhusudan Reddy (2010) has analyzed the effect of chemical reaction on double diffusive heat transfer flow of a viscous fluid in a wavy channel.

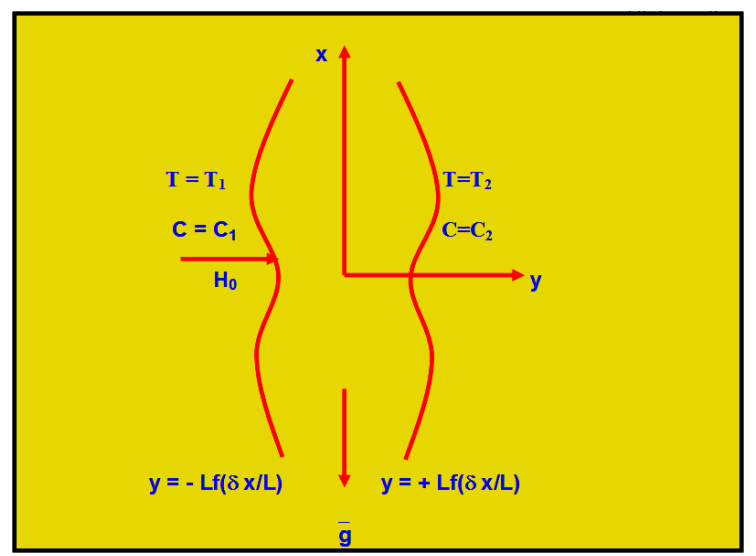

Fig. 1 Basic construction

\section{FORMULATION OF THE PROBLEM}

We consider the combined influence of radiation and chemical reaction on the unsteady motion of viscous, incompressible fluid through a porous medium in a vertical channel bounded by wavy walls. The thermal buoyancy in the flow field is created by an oscillatory flux in the fluid region. The walls are maintained at constant temperature and concentration. The Boussinesq approximation is used so that the density variation will be considered only in the buoyancy force. The viscous and Darcy dissipations are neglected in comparison with heat by conduction and convection in the energy equation. Also the Kinematic viscosity $v$, the thermal conducting $\mathrm{k}$ are treated as constants. We choose a rectangular Cartesian system $\mathrm{O}(\mathrm{x}, \mathrm{y})$ with $\mathrm{x}$-axis in the vertical direction and $y$-axis normal to the walls. The walls of the channel are at $y= \pm L f\left(\frac{\delta x}{L}\right)$ are:

The equations governing the unsteady flow, heat and mass transfer

Equation of continuity

$$
\frac{\partial u}{\partial x}+\frac{\partial v}{\partial y}=0
$$

Equation of linear momentum

$$
\begin{aligned}
& \rho_{e}\left(\frac{\partial u}{\partial t}+u \frac{\partial u}{\partial x}+v \frac{\partial u}{\partial y}\right)=-\frac{\partial p}{\partial x}+\mu\left(\frac{\partial^{2} u}{\partial x^{2}}+\frac{\partial^{2} u}{\partial y^{2}}\right)-\rho g-\left(\sigma \mu_{e}^{2} H_{o}^{2}\right) u-\left(\frac{\mu}{k}\right) u \\
& \rho_{e}\left(\frac{\partial v}{\partial t}+u \frac{\partial v}{\partial x}+v \frac{\partial v}{\partial y}\right)=-\frac{\partial p}{\partial y}+\mu\left(\frac{\partial^{2} v}{\partial x^{2}}+\frac{\partial^{2} v}{\partial y^{2}}\right)-\left(\frac{\mu}{k}\right) v
\end{aligned}
$$

Equation of Energy:

$$
\rho_{e} C_{p}\left(\frac{\partial T}{\partial t}+u \frac{\partial T}{\partial x}+v \frac{\partial T}{\partial y}\right)=\lambda\left(\frac{\partial^{2} T}{\partial x^{2}}+\frac{\partial^{2} T}{\partial y^{2}}\right)-Q\left(T-T_{e}\right)-\frac{\partial\left(q_{R}\right)}{\partial y}
$$

Equation of diffusion

$$
\left(\frac{\partial C}{\partial t}+u \frac{\partial C}{\partial x}+v \frac{\partial C}{\partial y}\right)=D_{1}\left(\frac{\partial^{2} C}{\partial x^{2}}+\frac{\partial^{2} C}{\partial y^{2}}\right)-k_{1}\left(C-C_{e}\right)
$$

Equation of state

$$
\rho-\rho_{e}=-\beta \rho_{e}\left(T-T_{e}\right)-\beta^{*} \rho_{e}\left(C-C_{e}\right)
$$

where $\rho_{e}$ is the density of the fluid in the equilibrium state, Te, Ce are the temperature and concentration in the equilibrium state, $(\mathrm{u}, \mathrm{v})$ are the velocity components along $\mathrm{O}(\mathrm{x}, \mathrm{y})$ directions, $\mathrm{p}$ is the pressure, $\mathrm{T}, \mathrm{C}$ are the temperature and Concentration in the flow region, $\rho$ is the density of the fluid, $\mu$ is the constant coefficient of viscosity, $\mathrm{Cp}$ is the specific heat at constant pressure, $\lambda$ is the coefficient of thermal conductivity, $\mathrm{k}$ is the permeability of the porous medium,$\beta$ is the coefficient of thermal expansion, $\mathrm{Q}$ is the strength of the constant internal heat source, $\sigma$ is the electrical conductivity , $\mu \mathrm{e}$ is the magnetic permeability, $\beta^{*}$ is the volumetric expansion with mass fraction coefficient $D_{1}$, is the molecular diffusivity and $\mathrm{k}_{1}$ is the chemical reaction coefficient.

In the equilibrium state

$$
0=-\frac{\partial p_{e}}{\partial x}-\rho_{e} g
$$

where $p=p_{e}+p_{D}, p_{D}$ being the hydrodynamic pressure.

The flow is maintained by an oscillatory volume flux for which a characteristic velocity is defined as

$$
q\left(1+k e^{i \omega t}\right)=\frac{1}{L} \int_{-L f}^{L f} u d y
$$

The boundary conditions for the velocity and temperature fields are

$$
\begin{array}{llc}
\mathrm{u}=0, \mathrm{v}=0, \mathrm{~T}=\mathrm{T} 1 & , \mathrm{C}=\mathrm{C} 1 & \text { on } \quad y=-L f\left(\frac{\delta x}{L}\right) \\
u=0, v=0, T=T_{2} & C=C_{2} \quad \text { on } & y=+L f\left(\frac{\delta x}{L}\right)
\end{array}
$$

In view of the continuity equation we define the stream function $\psi$ as

$$
\mathrm{u}=-\psi \mathrm{y}, \mathrm{v}=\psi \mathrm{x}
$$

Eliminating pressure $\mathrm{p}$ from equations (2.2)\&(2.3)and using the equations governing the flow in terms of $\psi$ are

$$
\begin{gathered}
{\left[\left(\nabla^{2} \psi\right)_{t}+\psi_{x}\left(\nabla^{2} \psi\right)_{y}-\psi_{y}\left(\nabla^{2} \psi\right)_{x}\right]=\nu \nabla^{4} \psi-\beta g\left(T-T_{0}\right)_{y}-} \\
\beta^{*} g\left(C-C_{0}\right)_{y}-\left(\sigma \mu_{e}^{2} H_{o}^{2}\right) \frac{\partial^{2} \psi}{\partial y^{2}}-\left(\frac{\mu}{k}\right) \nabla^{2} \psi \\
\rho_{e} C_{p}\left(\frac{\partial T}{\partial t}+\frac{\partial \psi}{\partial y} \frac{\partial T}{\partial x}-\frac{\partial \psi}{\partial x} \frac{\partial T}{\partial y}\right)=\lambda \nabla^{2} T-Q\left(T-T_{o}\right)+\frac{16 \sigma^{*} T_{e}^{3}}{\beta_{R}} \frac{\partial^{2} T}{\partial y^{2}} \\
\left(\frac{\partial C}{\partial t}+\frac{\partial \psi}{\partial y} \frac{\partial C}{\partial x}-\frac{\partial \psi}{\partial x} \frac{\partial C}{\partial y}\right)=D \nabla^{2} C-k_{1}\left(C-C_{o}\right)
\end{gathered}
$$

Introducing the non-dimensional variables

$$
x^{\prime}=x / L, y^{\prime}=y / L, t^{\prime}=t \omega, \Psi^{\prime}=\Psi / q L, \theta=\frac{T-T_{2}}{T_{1}-T_{2}}, C^{\prime}=\frac{C-C_{2}}{C_{1}-c_{2}}
$$

the governing equations in the non-dimensional form (after dropping the dashes ) are

$R\left(\gamma^{2}\left(\nabla^{2} \psi\right)_{t}+\frac{\partial\left(\psi, \nabla^{2} \psi\right)}{\partial(x, y)}\right)=\nabla^{4} \psi+\left(\frac{G}{R}\right)\left(\theta_{y}+N C_{y}\right)-M^{2} \frac{\partial^{2} \psi}{\partial y^{2}}-D^{-1} \nabla^{2} \psi$ 


$$
\begin{aligned}
& P\left(\gamma^{2} \frac{\partial \theta}{\partial t}+\frac{\partial \psi}{\partial y} \frac{\partial \theta}{\partial x}-\frac{\partial \psi}{\partial x} \frac{\partial \theta}{\partial y}\right)=\nabla^{2} \theta-\alpha \theta+\frac{4}{3 N_{1}} \frac{\partial^{2} \theta}{\partial y^{2}} \\
& S c\left(\gamma^{2} \frac{\partial C}{\partial t}+\frac{\partial \psi}{\partial y} \frac{\partial C}{\partial x}-\frac{\partial \psi}{\partial x} \frac{\partial C}{\partial y}\right)=\nabla^{2} C-K C
\end{aligned}
$$

The corresponding boundary conditions are $\psi(+f)-\psi(-f)=1$

$$
\begin{aligned}
& \frac{\partial \psi}{\partial x}=0, \quad \frac{\partial \psi}{\partial y}=0 \quad \text { aty } \eta= \pm f \\
& \theta(x, y)=1, C=1 \quad \text { on } \quad y=-f \\
& \theta(x, y)=0, C=0 \quad \text { on } \quad y=f \\
& \frac{\partial \theta}{\partial y}=0, \frac{\partial C}{\partial y}=0 \quad \text { at } \quad y=0
\end{aligned}
$$

The value of $\psi$ on the boundary assumes the constant volumetric flow in consistent with the hypothesis (7) .Also the wall temperature varies in the axial direction in accordance with the prescribed arbitrary function $\mathrm{t}$.

\section{METHOD OF SOLUTION}

The main aim of the analysis is to discuss the perturbations created over a combined free and forced convection flow due to traveling thermal wave imposed on the boundaries. The perturbation analysis is carried out by assuming that the aspect ratio $\delta$ to be small.

Introduce the transformation such that

$$
\bar{x}=\delta x, \frac{\partial}{\partial x}=\delta \frac{\partial}{\partial \bar{x}}
$$

Then

$$
\frac{\partial}{\partial x} \approx O(\delta) \rightarrow \frac{\partial}{\partial \bar{x}} \approx O(1)
$$

For small values of $\delta<<1$, the flow develops slowly with axial gradient of order $\delta$

And hence we take $\frac{\partial}{\partial \bar{x}} \approx O(1)$

Using the above transformation the equations (2.15-2.17) reduces to

$$
\delta R\left(\gamma^{2}\left(\nabla_{1}^{2} \psi\right)_{t}+\frac{\partial\left(\psi, \nabla_{1}^{2} \psi\right)}{\partial(x, y)}\right)=\nabla_{1}^{4} \psi-\left(\frac{G}{R}\right)\left(\theta_{y}+N C_{y}\right)-M^{2} \frac{\partial^{2} \psi}{\partial y^{2}}-D^{-1} \nabla_{1}^{2} \psi
$$$$
\delta P\left(\gamma^{2} \frac{\partial \theta}{\partial t}-\frac{\partial \psi}{\partial y} \frac{\partial \theta}{\partial x}+\frac{\partial \psi}{\partial x} \frac{\partial \theta}{\partial y}\right)=\nabla_{1}^{2} \theta-\alpha \theta
$$$$
\delta S c\left(\gamma^{2} \frac{\partial C}{\partial t}-\frac{\partial \psi}{\partial y} \frac{\partial C}{\partial x}+\frac{\partial \psi}{\partial x} \frac{\partial C}{\partial y}\right)=\nabla_{1}^{2} C-K C
$$

Where

$$
\nabla_{1}^{2}=\delta^{2} \frac{\partial^{2}}{\partial x^{2}}+\frac{\partial^{2}}{\partial y^{2}}
$$

Introducing the transformation

$$
\eta=\frac{y}{f(\bar{x})}
$$

the equations(3.1-3.3)reduces to

$$
\begin{gathered}
\delta R f\left(\gamma^{2}\left(F^{2} \psi\right)_{t}+\frac{\partial\left(\psi, F^{2} \psi\right)}{\partial(\bar{x}, \eta)}\right)=F^{4} \psi-\left(\frac{G f^{3}}{R}\right)\left(\theta_{\eta}+N C_{\eta}\right)- \\
\left(M^{2} f^{2}\right) \frac{\partial^{2} \psi}{\partial \eta^{2}}-D^{-1}\left(F^{2} \psi\right) \\
\delta P\left(\gamma^{2} \frac{\partial \theta}{\partial t}-f\left(\frac{\partial \psi}{\partial \eta} \frac{\partial \theta}{\partial x}+\frac{\partial \psi}{\partial x} \frac{\partial \theta}{\partial \eta}\right)=F^{2} \theta-\alpha f^{2} \theta\right. \\
\delta S c\left(\gamma^{2} \frac{\partial C}{\partial t}-f\left(\frac{\partial \psi}{\partial \eta} \frac{\partial C}{\partial x}+\frac{\partial \psi}{\partial x} \frac{\partial C}{\partial \eta}\right)=F^{2} C-K C\right.
\end{gathered}
$$

Where

$$
F^{2}=\delta^{2} \frac{\partial^{2}}{\partial \bar{x}^{2}}+\frac{\partial^{2}}{\partial \eta^{2}}
$$

We adopt the perturbation scheme and write $\psi(x, \eta, t)=\psi_{0}(x, \eta, t)+k e^{i t} \bar{\psi}_{0}(x, \eta, t)+\delta\left(\psi_{1}(x, \eta, t)+k e^{i t} . \bar{\psi}_{1}(x, \eta, t)\right)+\ldots \ldots .$. $\theta(x, \eta, t)=\theta_{0}(x, \eta, t)+k e^{i t} \bar{\theta}_{0}(x, \eta, t)+\delta\left(\theta_{1}(x, \eta, t)+k e^{i t} . \bar{\theta}_{1}(x, \eta, t)\right)+\ldots \ldots$. $C(x, \eta, t)=C_{0}(x, \eta, t)+k e^{i t} C \bar{\phi}_{0}(x, \eta, t)+\delta\left(C{ }_{1}(x, \eta, t)+k e^{i t} \cdot \bar{C}_{1}(x, \eta, t)\right)+\ldots \ldots$.

On substituting (3.1) in (3.4) - (3.6) and separating the like powers of $\delta$ the equations and respective conditions to the zeroth order are

$$
\begin{aligned}
& \psi_{0, \eta \eta \eta \eta}-\left(M^{2}+D^{-1}\right) \psi_{0, \eta \eta}=\left(\frac{G f^{3}}{R}\right)\left(\theta_{0, \eta}+N C_{0, \eta}\right) \\
& \theta_{0, \eta \eta}-\left(\alpha_{1} f^{2}\right) \theta_{0}=0 \\
& C_{0, \eta \eta}-\left(K S c f^{2}\right) C_{0}=0
\end{aligned}
$$

With

$$
\begin{aligned}
& \psi 0(+1)-\Psi 0(-1)=1, \\
& \psi 0, \eta=0, \psi 0, \mathrm{x}=0 \quad \text { at } \eta= \pm 1 \\
& \theta_{o}=1, \quad C_{0}=1 \quad \text { on } \quad \eta=-1 \\
& \theta_{o}=0, \quad C_{0}=0 \quad \text { on } \quad \eta=+1 \\
& \bar{\theta}_{0, \eta \eta}-\left(i P_{1} \gamma^{2} f^{2}\right) \bar{\theta}_{0}=0 \\
& \bar{C}_{0, \eta \eta}-\left(K S c \gamma^{2} f^{2}\right) \bar{C}_{o}=0 \\
& \bar{\psi}_{0, \eta \eta \eta \eta}-\left(\left(\left(M^{2}+D^{-1}\right)+i \gamma^{2}\right) f^{2}\right) \bar{\psi}_{0, \eta \eta}=\left(\frac{G f^{3}}{R}\right)\left(\bar{\theta}_{0, \eta}+N \bar{C}_{0, \eta}\right) \\
& \bar{\theta}_{o}( \pm 1)=0 \quad \bar{C}_{o}( \pm 1)=0 \\
& \bar{\psi}_{o}(+1)-\bar{\psi}_{o}(-1)=1 \quad \bar{\psi}_{o, \eta}( \pm 1)=0, \bar{\psi}_{o, x}( \pm 1)=0
\end{aligned}
$$

The first order equations are

$$
\begin{gathered}
\psi_{1, \eta \eta \eta \eta}-\left(\left(M^{2}+D^{-1}\right) f^{2}\right) \psi_{1, \eta \eta}=\left(\frac{G f^{3}}{R}\right)\left(\theta_{1, \eta}+N C_{1, \eta}\right)+(R f)\left(\psi_{0, \eta} \psi_{0, x \eta \eta}-\psi_{0, x} \psi_{0, \eta \eta \eta}\right) \\
\theta_{1, \eta \eta \eta}-\left(\alpha_{1} f^{2}\right) \theta_{1}=\left(P_{1} R f\right)\left(\psi_{0, x} \theta_{0, \eta}-\psi_{0, \eta} \theta_{0, x}\right) \\
C_{1, \eta \eta \eta}-\left(K S c f^{2}\right) C_{1}=(S c f)\left(\psi_{0, x} C_{0, \eta}-\psi_{0, \eta} C_{0, x}\right) \\
\bar{\psi}_{1, \eta \eta \eta \eta \eta}-\left(\left(\left(M^{2}+D^{-1}\right)+i \gamma^{2}\right) f^{2}\right) \bar{\psi}_{1, \eta \eta}=\left(\frac{G f^{3}}{R}\right)\left(\bar{\theta}_{1, \eta}+N \bar{C}_{1, \eta}\right)+(R f) \times \\
\left(\bar{\psi}_{0, \eta} \psi_{0, x \eta \eta}+\psi_{0, \eta} \bar{\psi}_{0, x \eta \eta}-\psi_{0, x} \bar{\psi}_{0, \eta \eta \eta}-\bar{\psi}_{0, x} \bar{\psi}_{0, \eta \eta \eta}\right) \\
\bar{\theta}_{1, \eta \eta \eta}-\left(\left(i P_{1} \gamma^{2}+\alpha_{1}\right) f^{2}\right) \bar{\theta}_{1}=\left(P_{1} R f\right)\left(\psi_{0, \eta} \bar{\theta}_{o, x}+\bar{\psi}_{0, \eta} \theta_{o x}-\bar{\psi}_{0, x} \theta_{o, \eta}-\psi_{0, x} \bar{\theta}_{o \eta}\right) \\
\bar{C}_{1, \eta \eta \eta}-\left(\left(K+i \gamma^{2}\right) S c f^{2}\right) \bar{C}_{1}=(S c f)\left(\psi_{0, \eta} \bar{C}_{o, x}+\bar{\psi}_{0, \eta} C_{o x}-\bar{\psi}_{0, x} C_{o, \eta}-\psi_{0, x} \bar{C}_{o \eta \eta}\right)
\end{gathered}
$$

with

$$
\begin{aligned}
& \psi 1(+1)-\psi 1(-1)=0 \\
& \psi 1, \eta=0, \psi 1, \mathrm{X}=0 \text { AT } \eta= \pm 1 \\
& \bar{\theta}_{1}( \pm 1)=0 \quad \bar{C}_{1}( \pm 1)=0 \\
& \bar{\psi}_{1}(+1)-\bar{\psi}_{1}(-1)=1 \quad \bar{\psi}_{1, \eta}( \pm 1)=0, \bar{\psi}_{1, x}( \pm 1)=0
\end{aligned}
$$

\section{SOLUTION OF THE PROBLEM}

Solving the equations (21) - (32) subject to the relevant boundary conditions we obtain

$$
\begin{aligned}
& C_{0}=0.5\left(\frac{C h\left(\beta_{1} \eta\right)}{\operatorname{Ch}\left(\beta_{1}\right)}-\frac{\operatorname{Sh}\left(\beta_{1} \eta\right)}{\operatorname{Sh}\left(\beta_{1}\right)}\right) \quad \theta_{0}=0.5\left(\frac{\operatorname{Ch} \beta_{2} \eta}{\operatorname{Ch} \beta_{2}}-\frac{\operatorname{Sh} \beta_{2} \eta}{\operatorname{Sh} \beta_{2}}\right) \\
& \psi_{o}=a_{13} \operatorname{Ch}\left(M_{1} \eta\right)+a_{14} \operatorname{Sh}\left(M_{1} \eta\right)+a_{15} \eta+a_{16}+f_{1}(\eta)
\end{aligned}
$$




$$
\begin{aligned}
& f_{1}(\eta)=a_{9} \operatorname{Sh}\left(\beta_{1} \eta\right)+a_{10} \operatorname{Ch}\left(\beta_{1} \eta\right)+a_{11} \operatorname{Sh}\left(\beta_{2} \eta\right)+a_{12} \operatorname{Ch}\left(\beta_{2} \eta\right) \\
& \bar{C}_{0}=0 \\
& \bar{\theta}_{0}=0 \\
& \bar{\psi}_{0}=a_{16} \operatorname{Sh}\left(M_{1} \eta\right)+a_{17} \eta+a_{18} \\
& C_{1}=a_{92} \operatorname{Ch}\left(\beta_{2} \eta\right)+a_{93} \operatorname{Sh}\left(\beta_{2} \eta\right)+f_{2}(\eta) \\
& f_{2}(\eta)=\left(a_{67}+\eta a_{79}\right) \operatorname{Ch}\left(\beta_{6} \eta\right)+\left(a_{68}+\eta a_{80}\right) \operatorname{Ch}\left(\beta_{7} \eta\right)+a_{69} \operatorname{Sh}\left(\beta_{6} \eta\right)+ \\
& +a_{70} \operatorname{Ch}\left(\beta_{7} \eta\right)+\left(a_{71}+\eta a_{83}\right) \operatorname{Sh}\left(\beta_{8} \eta\right)+a_{72} \operatorname{Sh}\left(\beta_{9} \eta\right)+ \\
& +\left(a_{73}+\eta a_{81}\right) \operatorname{Ch}\left(\beta_{8} \eta\right)+\left(a_{74}+\eta a_{82}\right) \operatorname{Ch}\left(\beta_{9} \eta\right)+ \\
& +\left(a_{75}+\eta\left(a_{78}+a_{88}\right)\right) \operatorname{Sh}\left(2 \beta_{1} \eta\right)+\left(a_{76}+\eta\left(a_{77}+a_{87}\right)\right) \operatorname{Ch}\left(2 \beta_{1} \eta\right)+ \\
& +a_{84} \eta \operatorname{Sh}\left(\beta_{5} \eta\right)+a_{85} \eta \operatorname{Sh}\left(\beta_{1} \eta\right)+a_{86} \eta \operatorname{Ch}\left(\beta_{1} \eta\right)+a_{89} \eta^{2} \operatorname{Ch}\left(\beta_{1} \eta\right)+ \\
& +a_{90} \eta^{2} \operatorname{Sh}\left(\beta_{1} \eta\right)-a_{91} \eta^{2} \\
& \theta_{1}=b_{92} \operatorname{Ch}\left(\beta_{1} \eta\right)+b_{93} \operatorname{Sh}\left(\beta_{1} \eta\right)+f_{3}(\eta) \\
& \beta_{1}^{2}=K S c f^{2}, \quad \beta_{2}^{2}=\alpha_{1} f^{2}, M_{1}^{2}=\left(M^{2}+D^{-1}\right) f^{2} \text {, } \\
& \beta_{3}=\beta_{1}+\beta_{2}, \beta_{4}=\beta_{1}-\beta_{2} \\
& f_{3}(\eta)=d_{1} \operatorname{Ch}\left(\beta_{10} \eta\right)+d_{2} \operatorname{Ch}\left(\beta_{11} \eta\right)+d_{3} \operatorname{Sh}\left(\beta_{10} \eta\right)+d_{4} \operatorname{Sh}\left(\beta_{11} \eta\right)+ \\
& +d_{5} \operatorname{Ch}\left(\beta_{9} \eta\right)+d_{6} \operatorname{Sh}\left(\beta_{9} \eta\right)+d_{7} \operatorname{Ch}\left(\beta_{8} \eta\right)+d_{8} \operatorname{Sh}\left(\beta_{8} \eta\right)+ \\
& +d_{9} \operatorname{Ch}\left(\beta_{7} \eta\right)+d_{10} \operatorname{Sh}\left(\beta_{7} \eta\right)+d_{11} \operatorname{Ch}\left(\beta_{6} \eta\right)+d_{12} \operatorname{Sh}\left(\beta_{6} \eta\right)+ \\
& +d_{13} \operatorname{Sh}\left(2 \beta_{1} \eta\right)+d_{14} \operatorname{Ch}\left(2 \beta_{1} \eta\right)+d_{15} \operatorname{Ch}\left(\beta_{2} \eta\right)+d_{16} \operatorname{Sh}\left(\beta_{2} \eta\right)+ \\
& +d_{17} \operatorname{Sh}\left(2 \beta_{2} \eta\right)+d_{18} \operatorname{Ch}\left(2 \beta_{2} \eta\right)+d_{19} \eta \operatorname{Ch}\left(\beta_{1} \eta\right)+d_{20} \eta \operatorname{Sh}\left(\beta_{1} \eta\right)+ \\
& +d_{21} \eta^{2} \operatorname{Ch}\left(\beta_{1} \eta\right)+d_{22} \eta^{2} \operatorname{Sh}\left(\beta_{1} \eta\right)+d_{23} \eta^{3} \operatorname{Ch}\left(\beta_{1} \eta\right)+d_{24} \eta^{3} \operatorname{Sh}\left(\beta_{1} \eta\right) \\
& +d_{25} \eta \operatorname{Sh}\left(2 \beta_{1} \eta\right)+d_{26} \eta \operatorname{Sh}\left(2 \beta_{2} \eta\right)+d_{27} \eta \operatorname{Ch}\left(2 \beta_{1} \eta\right)+d_{28} \eta \operatorname{Ch}\left(\beta_{2} \eta\right)+ \\
& +d_{29} \eta \operatorname{Sh}\left(\beta_{2} \eta\right)+d_{30} \eta \operatorname{Ch}\left(\beta_{2} \eta\right)+d_{31} \eta \operatorname{Ch}\left(\beta_{6} \eta\right)+d_{32} \eta \operatorname{Ch}\left(\beta_{7} \eta\right)+ \\
& +d_{33} \eta \operatorname{Ch}\left(\beta_{7} \eta\right)+d_{34} \eta \operatorname{Sh}\left(\beta_{8} \eta\right)+d_{35} \eta \operatorname{Sh}\left(\beta_{9} \eta\right)+d_{36} \eta \operatorname{Sh}\left(\beta_{6} \eta\right)+ \\
& +d_{37} \eta \operatorname{Sh}\left(\beta_{7} \eta\right)+d_{38} \eta \operatorname{Sh}\left(\beta_{10} \eta\right)+d_{39} \eta \operatorname{Sh}\left(\beta_{11} \eta\right)+d_{40} \eta \operatorname{Sh}\left(\beta_{11} \eta\right)+ \\
& +d_{41} \eta C h\left(\beta_{10} \eta\right)+d_{42} \eta C h\left(\beta_{11} \eta\right)-d_{43} \eta^{2}-d_{44} \\
& \psi_{1}=p_{1}+p_{2} \eta+p_{3} C h\left(M_{1} \eta\right)+p_{4} \operatorname{Sh}\left(M_{1} \eta\right)+f_{4}(\eta) \\
& f_{4}(\eta)=g_{1} \operatorname{Sh}\left(\beta_{1} \eta\right)+g_{2} \operatorname{Ch}\left(\beta_{1} \eta\right)+g_{3} \operatorname{Sh}\left(\beta_{2} \eta\right)+g_{4} \operatorname{Ch}\left(\beta_{2} \eta\right)+ \\
& +g_{5} \operatorname{Sh}\left(\beta_{6} \eta\right)+g_{6} \operatorname{Sh}\left(\beta_{7} \eta\right)+g_{7} \operatorname{Sh}\left(\beta_{8} \eta\right)+g_{8} \operatorname{Sh}\left(\beta_{9} \eta\right)+ \\
& +g_{9} \operatorname{Sh}\left(\beta_{10} \eta\right)+g_{10} \operatorname{Sh}\left(\beta_{11} \eta\right)+g_{11} \operatorname{Ch}\left(\beta_{6} \eta\right)+g_{12} \operatorname{Ch}\left(\beta_{7} \eta\right)+ \\
& +g_{13} C h\left(\beta_{8} \eta\right)+g_{14} C h\left(\beta_{9} \eta\right)+g_{15} C h\left(\beta_{10} \eta\right)+g_{16} C h\left(\beta_{11} \eta\right)+ \\
& +g_{17} C h\left(2 \beta_{1} \eta\right)+E_{97} \eta^{2} \operatorname{Sh}\left(M_{1} \eta\right)+E_{98} \eta^{2} C h\left(M_{1} \eta\right)+ \\
& +E_{99} \eta \operatorname{Sh}\left(M_{1} \eta\right)+E_{104} \eta \operatorname{Sh}\left(2 M_{1} \eta\right)+E_{105} \eta^{2} \operatorname{Sh}\left(2 M_{1} \eta\right)+ \\
& +E_{106} \operatorname{Ch}\left(2 M_{1} \eta\right)+a_{21} \operatorname{Sh}\left(\beta_{12} \eta\right)+a_{22} \operatorname{Sh}\left(\beta_{13} \eta\right)+a_{23} \operatorname{Sh}\left(\beta_{14} \eta\right)+ \\
& +a_{24} \operatorname{Sh}\left(\beta_{15} \eta\right)+a_{25} \operatorname{Ch}\left(\beta_{12} \eta\right)+a_{26} \operatorname{Ch}\left(\beta_{13} \eta\right)+a_{27} \operatorname{Ch}\left(\beta_{14} \eta\right)+ \\
& +a_{28} \operatorname{Ch}\left(\beta_{15} \eta\right)+E_{57} \eta^{3} \operatorname{Sh}\left(\beta_{1} \eta\right)+g_{29} \eta^{2} \operatorname{Sh}\left(\beta_{1} \eta_{-}\right)+ \\
& +g_{30} \eta \operatorname{Sh}\left(\beta_{1} \eta+E_{72} \eta^{3} \operatorname{Ch}\left(\beta_{1} \eta\right)+g_{31} \eta^{2} \operatorname{Ch}\left(\beta_{1} \eta\right)+g_{32} \eta \operatorname{Ch}\left(\beta_{1} \eta\right)\right.
\end{aligned}
$$

\section{NUSSELT NUMBER and SHERWOOD NUMBER}

The local rate of heat transfer coefficient (Nusselt number $\mathrm{Nu}$ ) on the walls has been calculated using the formula

where

$$
N u=\frac{1}{\theta_{m}-\theta_{w}}\left(\frac{\partial \theta}{\partial y}\right)_{\eta= \pm 1}
$$

$$
\theta_{m}=0.5 \int_{-1}^{1} \theta d \eta
$$

and the corresponding expressions are

$$
(N u)_{\eta=+1}=\frac{\left(d_{9}+\delta d_{11}\right)}{\left(\theta_{m}-\operatorname{Sin}(x+\gamma)\right)} ; \quad(N u)_{\eta=-1}=\frac{\left(d_{8}+\delta d_{10}\right)}{\left(\theta_{m}-1\right)}
$$

where

$\theta_{m}=d_{14}+\delta d_{15}$
The local rate of mass transfer coefficient (Sherwood Number Sh) on the walls has been calculated using the formula

$$
S h=\frac{1}{C_{m}-C_{w}}\left(\frac{\partial C}{\partial y}\right)_{y= \pm 1}
$$

where

$$
C_{m}=0.5 \int_{-1}^{1} C d y
$$

and the corresponding expressions are

$$
(S h)_{\eta=+1}=\frac{\left(d_{4}+\delta d_{6}\right)}{\left(C_{m}\right)} ; \quad(S h)_{\eta=-1}=\frac{\left(d_{5}+\delta d_{7}\right)}{\left(C_{m}-1\right)}
$$

where

$$
C_{m}=d_{12}+\delta d_{13}
$$

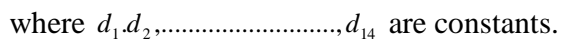

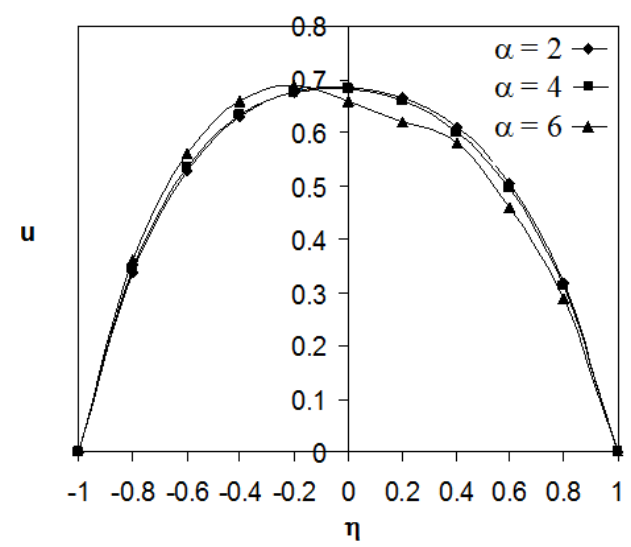

Fig. 1 Variation of $\mathrm{u}$ with $\alpha$

Figure 1 represents an increase in the strength of the heat source $(\alpha)$ larger $u$ in the left half and smaller in the right half of the channel. Figure 2 with respect to chemical reaction parameter $\mathrm{k}$ we find that the axial velocity experiences an enhancement in the left half and depreciates in the right half of the channel with increase in k. Fig. 3 represents an increase in the thermal radiation parameter $\mathrm{N} 1$, leads to an enhancement in the left half and depreciates in the right half of the channel. From Fig. 4 we find that higher the constriction of the channel walls lesser $u$ in the left half and larger in the right half of the channel.

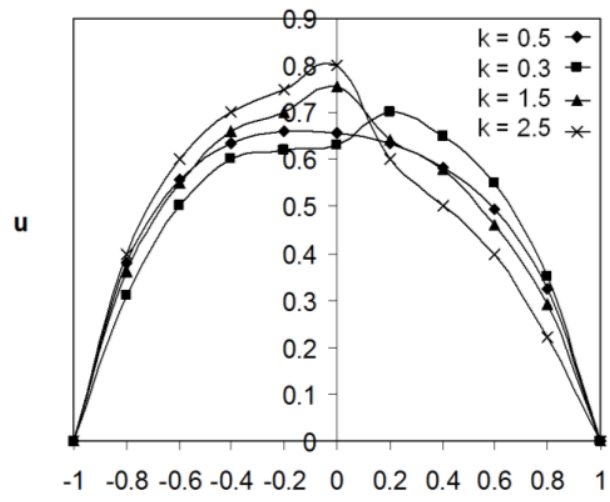

Fig. 2 Variation of $\mathrm{u}$ with $\mathrm{k}$ 


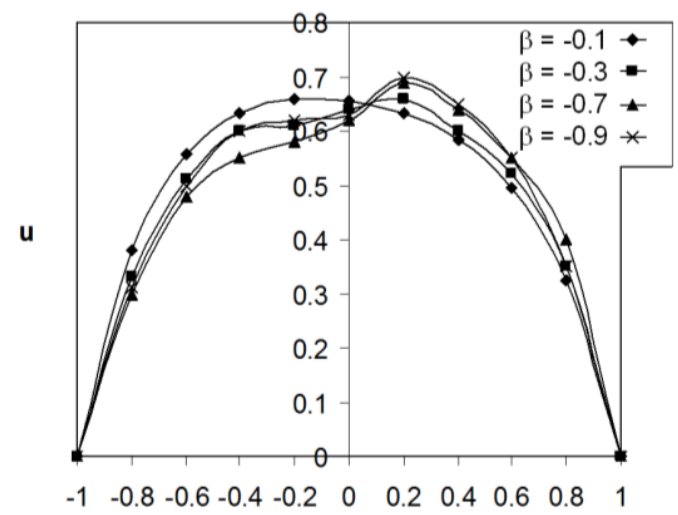

Fig. 3 Variation of $\mathrm{u}$ with $\beta$

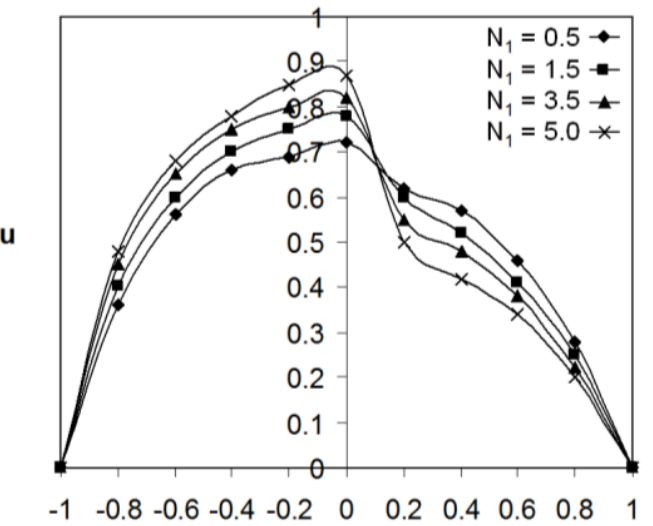

$\eta$

Fig. 4 Variation of $\mathrm{u}$ with $\mathrm{N} 1$

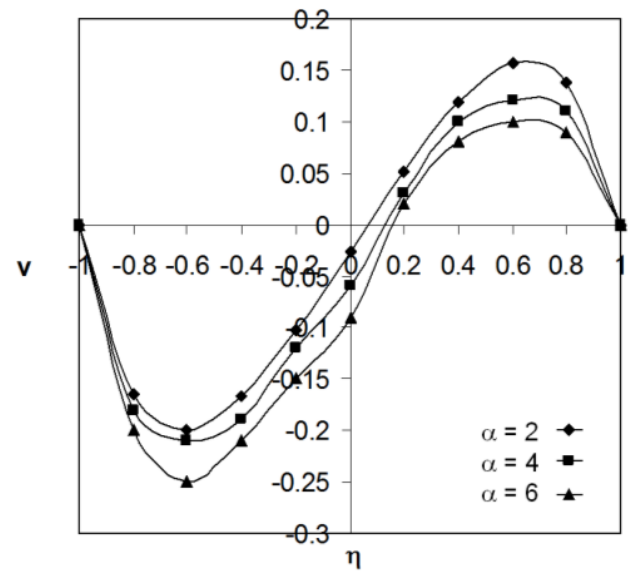

Fig. 5 Variation of $\mathrm{v}$ with $\alpha$

Figure 5-8 represents an increase in $\alpha$ enhances $|v|$ in the left half and reduces if in the right half of the channel. The magnitude of $\mathrm{v}$ enhances with increase in the chemical reaction parameter $\mathrm{k}$. An increase in $\mathrm{N} 1$ enhances $|\mathrm{v}|$ in the left half and depreciates in the right half. Higher the constriction of the channel walls larger $|\mathrm{v}|$ in the entire flow region. From Fig. 9-12 an increase in the chemical reaction parameter $k$ depreciates in the non-dimensional temperature, an increase in the chemical reaction parameter $\mathrm{k}$ depreciates in the non-dimensional temperature, the variation of $\theta$ with $\beta$ shows that higher the constriction of the channel walls larger the actual temperature, we find that higher the thermal radiative heat fluxes lesser the actual temperature in the flow region.

From Figs. 13-16, we find that the actual concentration depreciates with increase in the strength of the heat source. The variation of $\mathrm{C}$ with radiation parameter $\mathrm{N}_{1}$ shows that higher the thermal radiative heat flux lesser the actual concentration in the flow region. The effect of wall waviness on $\mathrm{C}$ is shown in. Higher the constriction of the channel walls lesser the actual concentration in the entire flow region. The variation of $\mathrm{C}$ with chemical reaction parameter $\mathrm{k}$ shows that the actual concentration enhances in the degenerating chemical reaction case.

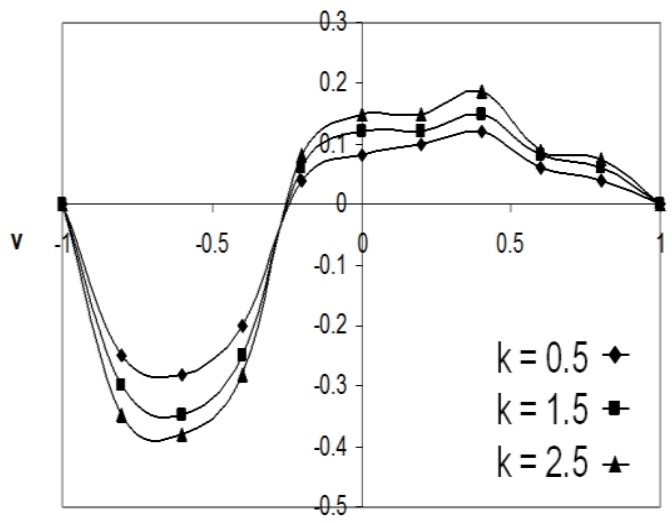

Fig. 6 Variation of $\mathrm{v}$ with $\mathrm{k}$

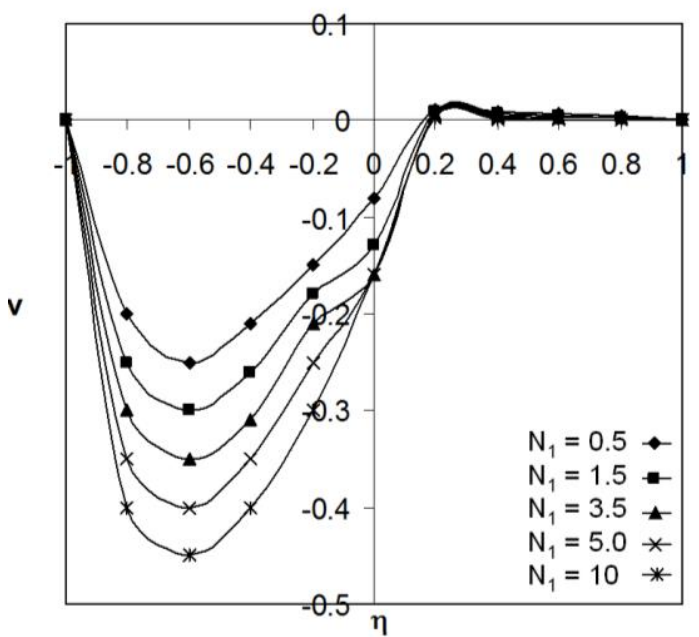

Fig. 7 Variation of $\mathrm{v}$ with $\mathrm{N}_{1}$

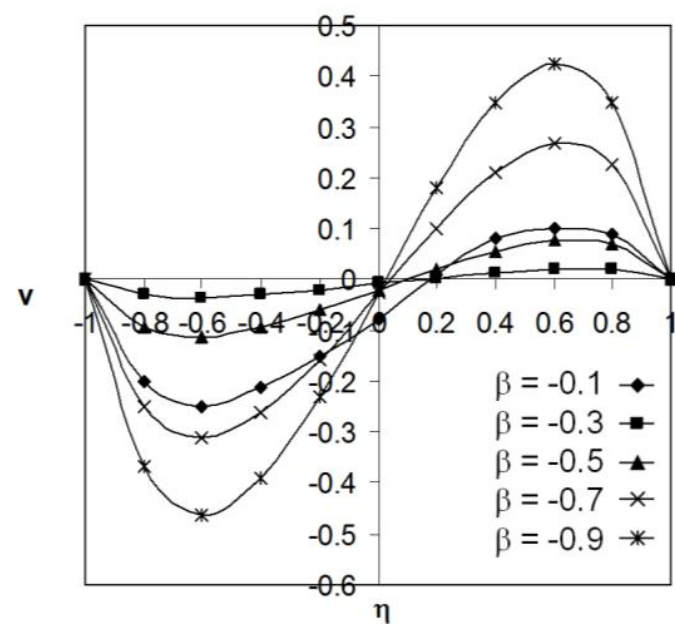

Fig. 8 Variation of $v$ with $\beta$ 


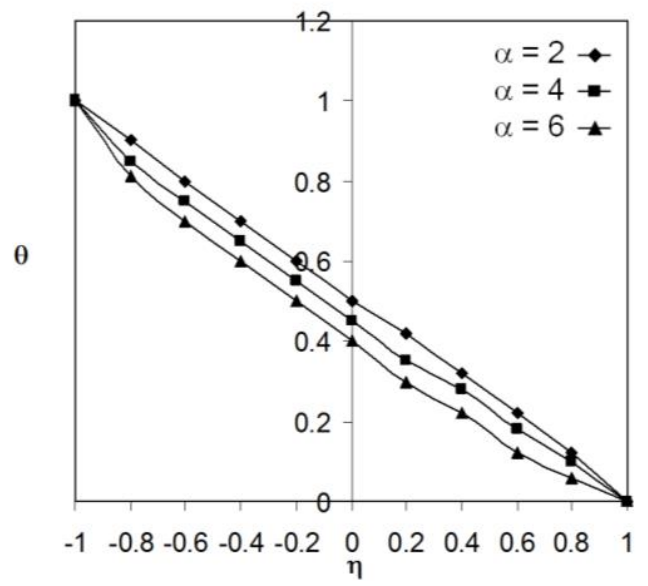

Fig. 9 Variation of $\theta$ with $\alpha$

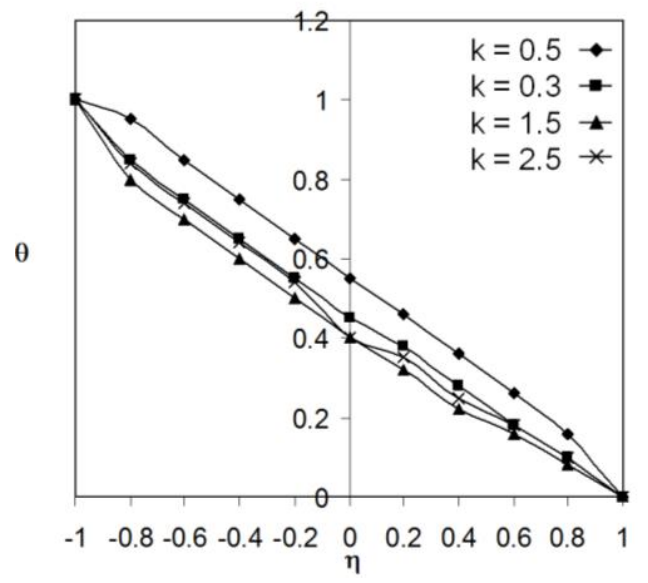

Fig. 10 Variation of $\theta$ with $\mathrm{k}$

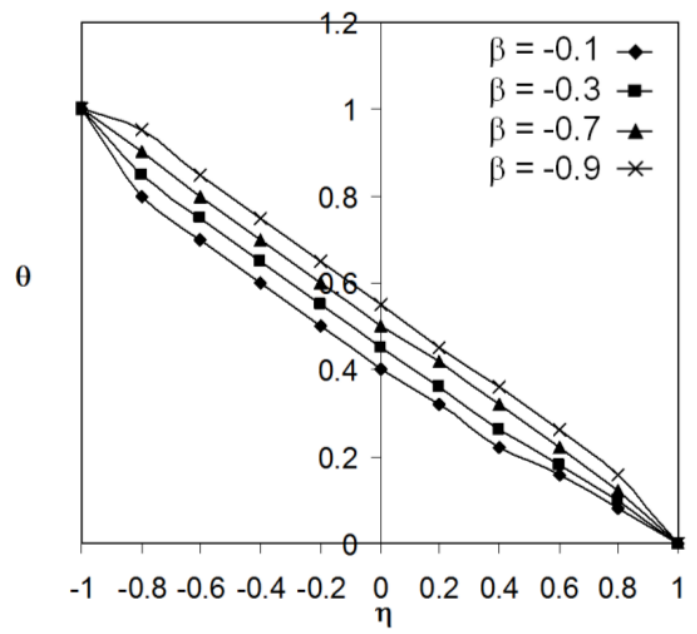

Fig. 11 Variation of $\theta$ with $\beta$

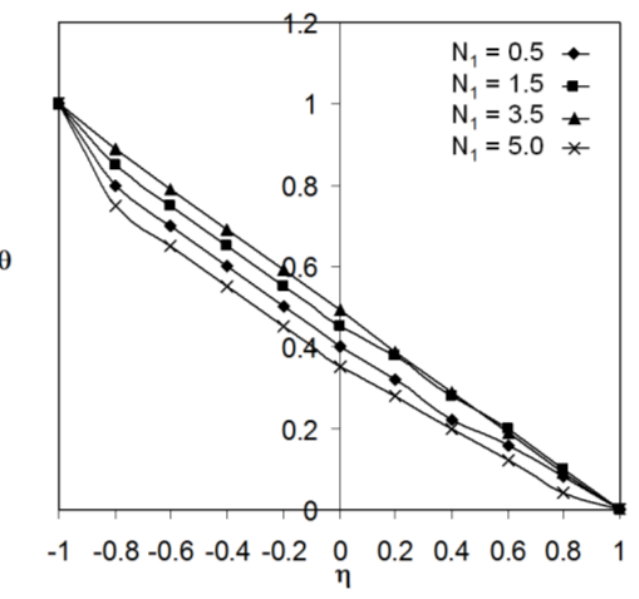

Fig. 12 Variation of $\theta$ with $\mathrm{N}_{1}$

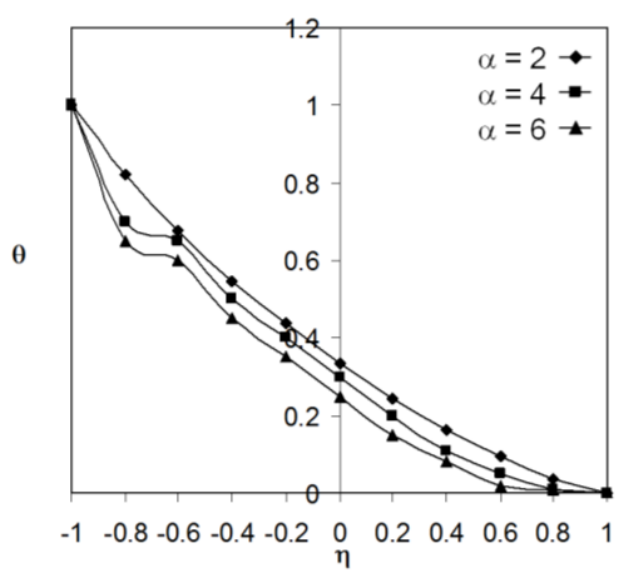

Fig. 13 Variation of $\mathrm{C}$ with $\alpha$

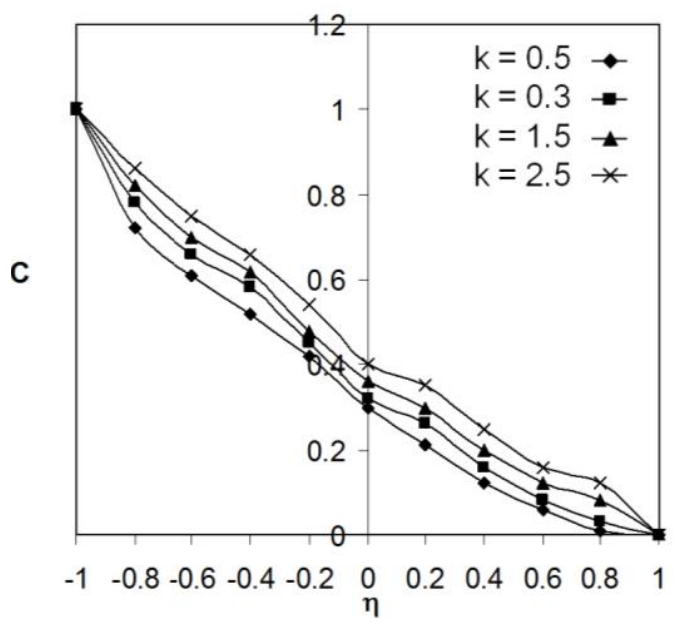

Fig. 14 Variation of $\mathrm{C}$ with $\mathrm{k}$ 


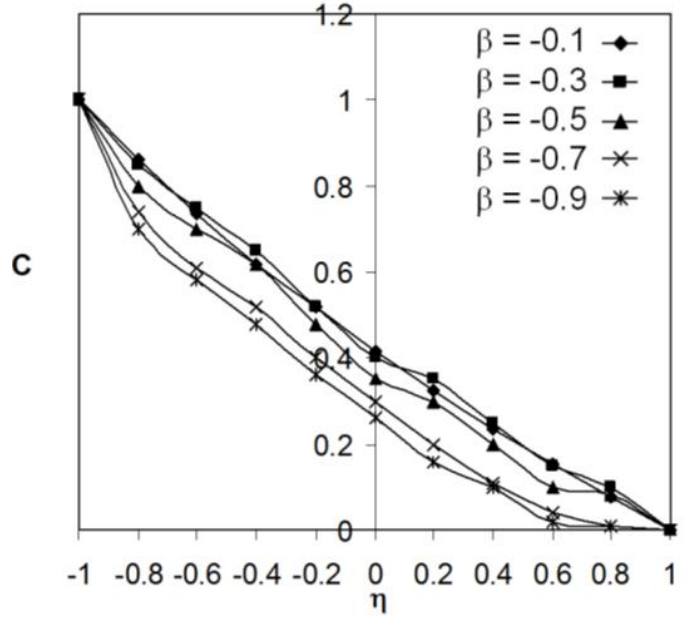

Fig. 15 Variation of $C$ with $\beta$

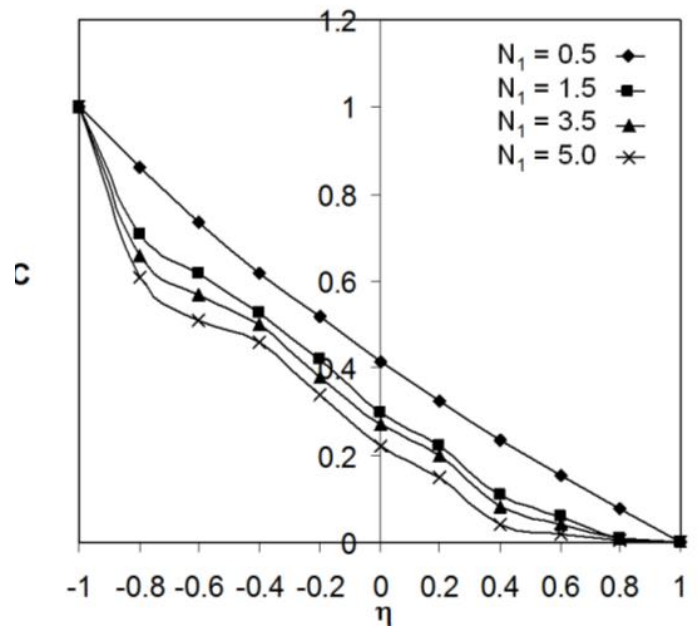

Fig. 16 Variation of $\mathrm{C}$ with $\mathrm{N}_{1}$

Table 1 Nusselt number $(\mathrm{Nu})$ at $\eta=+1$

\begin{tabular}{|c|c|c|c|c|c|c|c|}
\hline $10^{3}$ & $3 \times 10^{3}$ & $-10^{3}$ & $-3 \times 10^{3}$ & $K$ & $\alpha$ & $\beta$ & $N_{I}$ \\
\hline 1.12510 & 1.11642 & 1.13367 & 1.14229 & 0.5 & 2 & -0.5 & 0.5 \\
\hline 0.16797 & 0.13180 & 0.20412 & 0.24026 & 1.5 & 2 & -0.5 & 0.5 \\
\hline-0.01251 & -0.32169 & 0.29649 & 0.60531 & 2.5 & 2 & -0.5 & 0.5 \\
\hline 0.39464 & 0.39431 & 0.39496 & 0.39528 & 0.5 & 4 & -0.5 & 0.5 \\
\hline 0.33994 & 0.34368 & 0.33629 & 0.33275 & 0.5 & 6 & -0.5 & 0.5 \\
\hline 0.92106 & 0.91917 & 0.92295 & 0.92484 & 0.5 & 2 & -0.1 & 0.5 \\
\hline 1.01455 & 1.00879 & 1.02032 & 1.02608 & 0.5 & 2 & -0.3 & 0.5 \\
\hline 1.25697 & 1.24653 & 1.26740 & 1.27783 & 0.5 & 2 & -0.7 & 0.5 \\
\hline 1.40491 & 1.39405 & 1.41577 & 1.42662 & 0.5 & 2 & -0.9 & 0.5 \\
\hline 0.39984 & 0.39943 & 0.40024 & 0.40064 & 0.5 & 2 & -0.5 & 1.5 \\
\hline 0.35386 & 0.35487 & 0.35286 & 0.35187 & 0.5 & 2 & -0.5 & 3.5 \\
\hline 0.34369 & 0.34617 & 0.34126 & 0.33889 & 0.5 & 2 & -0.5 & 5.0 \\
\hline 0.33452 & 0.34615 & 0.32377 & 0.31380 & 0.5 & 2 & -0.5 & 10 \\
\hline
\end{tabular}

Table 2 Nusselt number $(\mathrm{Nu})$ at $\eta=-1$

\begin{tabular}{|c|c|c|c|c|c|c|c|}
\hline $10^{3}$ & $3 \times 10^{3}$ & $-10^{3}$ & $-3 \times 10^{3}$ & $K$ & $\alpha$ & $\beta$ & $N_{I}$ \\
\hline 3.21051 & 3.18767 & 3.23330 & 3.25610 & 0.5 & 2 & -0.5 & 0.5 \\
\hline 0.50714 & 0.39812 & 0.61601 & 0.72472 & 1.5 & 2 & -0.5 & 0.5 \\
\hline-0.03988 & -1.02613 & 0.94454 & 1.92711 & 2.5 & 2 & -0.5 & 0.5 \\
\hline 0.95354 & 0.95453 & 0.95255 & 0.95158 & 0.5 & 4 & -0.5 & 0.5 \\
\hline 0.74131 & 0.76254 & 0.72137 & 0.70259 & 0.5 & 6 & -0.5 & 0.5 \\
\hline 2.35598 & 2.35106 & 2.36091 & 2.36583 & 0.5 & 2 & -0.1 & 0.5 \\
\hline 2.73450 & 2.71980 & 2.74919 & 2.76387 & 0.5 & 2 & -0.3 & 0.5 \\
\hline 3.79387 & 3.76516 & 3.82252 & 3.85110 & 0.5 & 2 & -0.7 & 0.5 \\
\hline 4.32027 & 4.29045 & 4.35003 & 4.37970 & 0.5 & 2 & -0.9 & 0.5 \\
\hline 0.97356 & 0.97423 & 0.97289 & 0.97223 & 0.5 & 2 & -0.5 & 1.5 \\
\hline 0.79422 & 0.80163 & 0.78689 & 0.77991 & 0.5 & 2 & -0.5 & 3.5 \\
\hline 0.75523 & 0.77006 & 0.74108 & 0.72752 & 0.5 & 2 & -0.5 & 5.0 \\
\hline 0.72392 & 0.78617 & 0.67135 & 0.62657 & 0.5 & 2 & -0.5 & 10 \\
\hline
\end{tabular}

Table 3 Sherwood number (Sh) at $\eta=+1$

\begin{tabular}{|c|c|c|c|c|c|c|c|}
\hline $10^{3}$ & $3 \times 10^{3}$ & $-10^{3}$ & $-3 \times 10^{3}$ & $K$ & $\alpha$ & $\beta$ & $N_{I}$ \\
\hline-0.51272 & -0.52083 & -0.50466 & -0.49665 & 0.5 & 2 & -0.5 & 0.5 \\
\hline-0.42606 & -0.43299 & -0.41920 & -0.41240 & 1.5 & 2 & -0.5 & 0.5 \\
\hline-0.36111 & -0.38803 & -0.33489 & -0.30846 & 2.5 & 2 & -0.5 & 0.5 \\
\hline-0.74539 & -0.75093 & -0.74093 & -0.73595 & 0.5 & 4 & -0.5 & 0.5 \\
\hline-0.96130 & -0.96352 & -0.95903 & -0.95673 & 0.5 & 6 & -0.5 & 0.5 \\
\hline-0.21249 & -0.21266 & -0.21232 & -0.21215 & 0.5 & 2 & -0.1 & 0.5 \\
\hline-0.36184 & -0.36533 & -0.35837 & -0.35491 & 0.5 & 2 & -0.3 & 0.5 \\
\hline-0.69188 & -0.70543 & -0.67840 & -0.66501 & 0.5 & 2 & -0.7 & 0.5 \\
\hline-0.94482 & -0.96493 & -0.92483 & -0.90497 & 0.5 & 2 & -0.9 & 0.5 \\
\hline-0.73347 & -0.73864 & -0.72831 & -0.72317 & 0.5 & 2 & -0.5 & 1.5 \\
\hline-0.88459 & -0.88777 & -0.88140 & -0.87818 & 0.5 & 2 & -0.5 & 3.5 \\
\hline-0.93738 & -0.93989 & -0.93483 & -0.93225 & 0.5 & 2 & -0.5 & 5.0 \\
\hline-1.01688 & -1.01846 & -1.01524 & -1.01354 & 0.5 & 2 & -0.5 & 10 \\
\hline
\end{tabular}

Table 4 Sherwood number (Sh) at $\eta=-1$

\begin{tabular}{|c|c|c|c|c|c|c|c|}
\hline $10^{3}$ & $3 \times 10^{3}$ & $-10^{3}$ & $-3 \times 10^{3}$ & $K$ & $\alpha$ & $\beta$ & $N_{l}$ \\
\hline-3.58679 & -3.57838 & -3.59524 & -3.60376 & 0.5 & 2 & -0.5 & 0.5 \\
\hline-3.28566 & -3.26364 & -3.30806 & -3.33083 & 1.5 & 2 & -0.5 & 0.5 \\
\hline-3.06827 & -3.00708 & -3.13091 & -3.19501 & 2.5 & 2 & -0.5 & 0.5 \\
\hline-5.96445 & -5.93697 & -5.999192 & -6.01936 & 0.5 & 4 & -0.5 & 0.5 \\
\hline-16.90677 & -11.78356 & -12.02938 & -12.15175 & 0.5 & 6 & -0.5 & 0.5 \\
\hline-1.87421 & -1.87371 & -1.8747 & -1.87518 & 0.5 & 2 & -0.1 & 0.5 \\
\hline-2.59156 & -2.58743 & -2.5957 & -2.59984 & 0.5 & 2 & -0.3 & 0.5 \\
\hline-5.21615 & -5.20036 & -5.23212 & -5.24826 & 0.5 & 2 & -0.7 & 0.5 \\
\hline-8.62135 & -8.57364 & -8.66998 & -8.71963 & 0.5 & 2 & -0.9 & 0.5 \\
\hline-5.7786 & -5.75304 & -5.80416 & -5.82971 & 0.5 & 2 & -0.5 & 1.5 \\
\hline-8.94114 & -8.87494 & -9.00802 & -9.07465 & 0.5 & 2 & -0.5 & 3.5 \\
\hline-10.81453 & 10.713998 & -10.91478 & -11.01467 & 0.5 & 2 & -0.5 & 5 \\
\hline-15.40237 & -15.19252 & -15.61235 & -15.8223 & 0.5 & 2 & -0.5 & 10 \\
\hline
\end{tabular}

\section{CONCLUSIONS}

With respect to chemical reaction parameter $\mathrm{k}$ we find that the axial velocity experiences an enhancement in the left half and depreciates in the right half of the channel with increase in $\mathrm{k}$. The magnitude of $\mathrm{v}$ enhances with increase in the chemical reaction parameter $\mathrm{k}$. An increase in the chemical reaction parameter $\mathrm{k}$ reduces the actual temperature and enhances the actual concentration in the degenerating chemical reaction case.

An increase in the chemical reaction parameter $\mathrm{k}$ reduces $|\mathrm{Nu}|$ at $\eta$ $=+1$ while at $\eta=-1$, it reduces with $\mathrm{k} \leq 1.5$ and enhances with higher $\mathrm{k} \geq 2.5$ while $|\mathrm{Sh}|$ reduces with increase in $\mathrm{k}$. 
An increase in the strength of the heat source $(\alpha)$, larger $\mathrm{u},|\mathrm{v}|$ in the left half and smaller in the right half of the channel and leads to a depreciation in the actual temperature and the actual concentration in the entire flow region.

An increase in the strength of the heat source leads to a depreciation in $|\mathrm{Nu}|$ and enhancement in $|\mathrm{Sh}|$ at both the walls.

An increase in the thermal radiation parameter $\mathrm{N}_{1}$, leads to an enhancement in $\mathrm{u},|\mathrm{v}|$ in the left half and depreciates in the right half of the channel. Higher the thermal radioactive heat flux lesser the actual temperature and the actual concentration in the flow region.

Higher the thermal radioactive heat fluxes smaller the rate of heat transfer at both the walls.

Higher the constriction of the channel walls lesser $\mathrm{u}$ in the left half and larger in the right half of the channel. Higher the constriction of the channel walls larger $|v|, \theta$ and lesser the actual concentration in the entire flow region.

Higher the constriction of the channel walls larger $|\mathrm{Nu}|,|\mathrm{Sh}|$ at $\eta=$ \pm 1 .

\section{NOMENCLATURE}

$\begin{array}{ll}\mathrm{Re} & \text { Reynolds Number }\left(\frac{q L}{v}\right) \\ \mathrm{Gr} & \text { Grashof number }\left(\frac{\beta g \Delta L^{2} T_{e}}{v^{2}}\right) \\ \mathrm{Pr} & \text { Prandtl Number }\left(\frac{\mu C_{p}}{K_{1}}\right) \\ D a^{-1} & \text { Inverse Darcy Parameter }\left(\frac{L^{2}}{k}\right) \\ \mathrm{M}^{2} & \text { Hartmann Number }\left(\frac{\sigma L^{2} H_{0}^{2} \mu_{e}^{2}}{v^{2}}\right) \\ \mathrm{S}_{\mathrm{c}} & \text { Schmidt Number }\left(\frac{v}{D_{1}}\right) \\ \mathrm{A} & \text { Heat source parameter }\left(\frac{Q L^{2}}{\lambda}\right) \\ \mathrm{K} & \text { Chemical reaction parameter }\left(\frac{K_{1} L^{2}}{D_{1}}\right) \\ \tau^{2} & \text { Womersley number }\left(\frac{\omega L^{2}}{v}\right) \\ \mathrm{N}_{1} & \text { Radiation Parameter }\left(\frac{\lambda \beta_{R}}{4 T_{e}^{3} \sigma^{*}}\right)\end{array}$

\section{REFERENCES}

Angirasaa ,D., Peterson, G.P., and Pop. I., 1997, “Combined Heat and Mass Transfer by Natural Convection with Opposing Buoyancy Effects in a Fluid Saturated Porous Medium," International Journal of Heat and Mass Transfer, 40, 2755-2773.

http://dx.doi.org/10.1016/S0017-9310(96)00354-7

Bejan, A., and Khair, K.R., 1985, "Heat and Mass Transfer by Natural Convection in a Porous Medium," International Journal of Heat \& Mass Transfer, 28, 908-918. http://dx.doi.org/10.1016/0017-9310(85)90272-8

Chamkha, A,J., Takhar, H.S., and Soundalgekar, V.M., 2001, "Radiation Effects on Free Convection Flow Past a Semi-infinite Vertical Plate with Mass Transfer," Chemical Engineering Journal, 84, 335-342. http://dx.doi.org/10.1016/S1385-8947(00)00378-8

Comini. G. , Nonino. C., and Savino. S., 2002, "Convective Heat and Mass Transfer in Wavy Finned-tube Exchangers," International journals of Numerical Methods for Heat and Fluid flow, 12, 735-755. http://dx.doi.org/10.1108/09615530210438373

Cheng. P., 1978, "Heat Transfer in Geothermal Systems," Advances in Heat Transfer, 14, 1-105

\section{http://dx.doi.org/10.1016/S0065-2717(08)70085-6}

Deshikachar, K.S., and Ramachandra Rao, A., 1985, "Effect of a Magnetic Field on the Flow and Blood Oxygenation in Channel of Variable Cross-section," International journal of Engineering Science, 23, 1121.

http://dx.doi.org/10.1016/0020-7225(85)90034-5

Das, U.N., Deka, R.K., and Soundalgekar, V.M., 1994, "Effect of Mass Transfer on Flow Past an Impulsively Started Infinite Vertical Plate with Constant Heat Flux and Chemical Reaction," Forschung in Ingenierwegen, 60, 284-287. http://dx.doi.org/10.1007/BF02601318

Gagan, S., 1985, "Proceedings of Natural Heat and Mass Transfer Conference," Visakhapatnam, India.

Gnaneswar reddy., 2008, "Radiation and Mass Transfer on an Unsteady Two-Dimensional Laminar Convective Boundary Layer Flow of a Viscous Incompressible Chemically Reacting Fluid," Acta Ciencia Indica, 34, 639.

Hussain, M.A., and Takhar, H,S., 1996, "Radiation Effects on Mixed Convection along a Vertical Plate with Uniform Surface Temperature," Heat and Mass Transfer, 31, 243-248. http://dx.doi.org/10.1007/BF02328616

Kwon, H.G., Hwang, S.D., Cho, H.H., 2008, "Flow and Heat/Mass Transfer in a Wavy Duct with Various Corrugation Angles in Two Dimensional Flow Regimes," Heat and Mass Transfer, 45, 157-165. http://dx.doi.org/10.1007/s00231-008-0422-4

Lai, F.C., 1971, "Coupled Heat and Mass Transfer by Mixed Convection from a Vertical Plate in a Saturated Porous Medium," International Communications in Heat and Mass Transfer, 18, 93-106. http://dx.doi.org/10.1016/0735-1933(91)90011-R

Lai, F.C., and Kulacki, F.A., 1991, "Coupled Heat and Mass Transfer by Natural Convection from Vertical Surfaces in Porous Medium," International Journal of Heat \& Mass Transfer, 34, 1189-1194. http://dx.doi.org/10.1016/0017-9310(91)90027-C

Kandaswamy, P., Abdul Wahid, B., Md. Raj., B. Khamis., 2006, "Effects of Chemical Reaction, Heat and Mass Transfer on Boundary Layer Flow over a Porous Wedge with Heat Radiation in the Presence of Suction or Injection," Theoretical and Applied Mechanics., 33, 123-148. http://dx.doi.org/10.2298/TAM0602123K

Lee, T.S., Parikh, P.G., Archivos, A., and Bershader, D., 1982, "Natural Convection in a Vertical Channel with Opposing Buoyancy Forces," International Journal of Heat and Mass Transfer, 25, 499-522. http://dx.doi.org/10.1016/0017-9310(82)90053-9

McMichael, M., and Deutsch, S., 1984, "Magneto Hydrodynamics of Laminar Flow in Slowly Varying Tube in an Axial Magnetic Field," Physics of Fluids, 27, 110-118.

Mabunni. R., U. Rajeswar Rao., D.R.V. Prasada Rao., 2014, “Transient Convective Heat and Mass Transfer Flow of a Chemically Reacting Viscous Fluid in a Vertical Wavy Channel with Oscillatory Flux with Heat Sources," International Journal of Emerging Trends in Engineering and Development, 4, 137-150.

Madhusudan Reddy, Y., 2010, "Mixed Convective Heat and Mass Transfer Through a Porous Medium in Channels with Radiation Effect," Ph.D. thesis, S.K.University, Anantapur, India.

Murthy, P.V.S.N., and Singh. P., 1990, "Heat and Mass Transfer by Natural Convection in a Non-Darcy Porous Medium," Acta Mech, 26, 567.

http://dx.doi.org/10.1007/BF01291847 
Muthukumaraswamy, R., and Ganesan, P., 2003, International Journal of Applied Mechanics and Engineering, 44, 104.

Muthukumaraswamy, R., 2002, "Effects of a Chemical Reaction on a Moving Isothermal Surface with Suction," Acta Mechanica, 155, 65-72. http://dx.doi.org/10.1007/BF01170840

Nelson, D.J., and Wood, B.D., 1986, "Combined Heat and Mass Transfer by Natural Convection between Vertical Plates with Uniform Flux Boundary Conditions," Heat Transfer, 4, 1587-1592.

Nelson, D.J., and Wood, B.D., 1989, “Combined Heat and Mass Transfer by Natural Convection between Vertical Plates," International Journal of Heat \& Mass Transfer, 82, 1789-1792. http://dx.doi.org/10.1016/0017-9310(89)90059-8

Prakash, J., and Ogulu., 2006, "Unsteady Two-Dimensional Flow of a Radiating and Chemically Reacting MHD Fluid with Time-Dependent Suction," Indian Journal of Pure and Applied Maths, 44, 805-810.

Prasad, V., Kulacku, F.A., and Keyhani, M., 1985, "Natural Convection in a Porous Medium," Journal of Fluid Mech., 150, 89-119.

Prasada Rao. D.R.V,, Krishna, D.V., and Debnath, L., 1983, "Free Convection in Hydro Magnetic Flows in a Vertical Wavy Channel," International journal of Engineering Sciences, 21, No.9, 1025-1039.

Raptis, A., and Perdikis, C., 1999, "Radiation and Free Convection Flow Past a Moving Plate," Journal of Applied Mechanical Engineering, 4,817-821.

Soundalgekar, V.M., and Takhar, H.S., 1993, "Radiation Effects on Free Convection Flow past a Semi-Infinite Vertical Plate," Modeling Measurement and Control, 51, 31-40.

Sree Ramachandra Murthy, A., 1992, "Buoyancy Induced Hydro Magnetic Flows through a Porous Medium - A Study," Ph.D. thesis, S.K.University, Anantapur, India.

Sparrow, E.M., Chryser, M., and Azvedo, L.F.A., 1984, “'Observed Flow Reversals and Measured-Predicted Nusselt Numbers for Natural
Convection in a One-Sided Heated Vertical Channel," Journal of Heat Transfer, 106, 325-332.

Sparrow, E.M., and Azvedo, L.F.A., 1985, "Vertical-Channel Natural Convection Spanning between the Fully-Developed Limit and the Single-Plate Boundary-Layer Limit" International Journal of Heat and Mass Transfer, 28, 1847-1857. http://dx.doi.org/10.1016/0017-9310(85)90207-8

Sudha Mathew., 2009, "Hydro Magnetic Mixed Convective Heat and Mass Transfer Through a Porous Medium in a Vertical Channel with Thermo-diffusion Effect," Ph.D thesis, S.K. University, Anantapur, India.

Trevison, D.V., and Bejan, A., 1987, "Combined Heat and Mass Transfer by Natural Convection in Vertical Enclosure," Trans. ASME, 109, 104111.

Vajravelu K., and Ali Neyfeh., 1981, "Influence of Wall Waviness on Friction and Pressure Drop in Channels," International Journal of Mathematics \& Mathematical Sciences, 4, 805-818. http://dx.doi.org/10.1155/S0161171281000628

Vajravelu, K., and Sastry, K.S., 1978, "Heat Transfer in a Viscous Incompressible Fluid Confined Between a Long Vertical Wavy Wall and a Parallel Flat Wall," Journal of Fluid Mech., 86, 365. http://dx.doi.org/10.1017/S0022112078001172

Vajravelu, K., and Debnath, L., "Non-linear Study of Convective Heat Transfer and Fluid Flows Induced by Traveling Thermal Waves," Acta Mechanica, 59, 233-249.

Yan, W.M., 1996, “Combined Buoyancy Effects of Thermal and Mass Diffusion on Laminar Forced Convection in Horizontal Rectangular Ducts," International Journal of Heat \& Mass Transfer, 39, 1479-1488. http://dx.doi.org/10.1016/0017-9310(95)00227-8 